\title{
CAE modeling rubber-metal body mounting of the body-on-frame car in crash simulations
}

\author{
V.A. Tsittser, S.V. Alekseev, A.V. Tarasov, A.I. Borovkov, \\ Peter the Great St. Petersburg Polytechnic University (SPbPU), \\ 195251, St. Petersburg \\ Russia
}

Received: March 13, 2020. Revised: May 14, 2020. 2nd Revised: June 26, 2020. Accepted: June 29, 2020. Published: June 30, 2020

\begin{abstract}
The aim of the study was to research the behavior of the rubber-metal body mounting under various modeling options and to select the optimal, from the point of view of ensuring the accuracy of the results in the crash tests simulations. Body supports provide a link between the body and the car frame, and this has a critical effect on the impact test results of the car. The article discusses various options for modeling the body mounting by the degree of simplification from the simplest model with a rigid connection between the body and the frame to the model that takes into account the non-linearity of the stiffness characteristics of the supports, contact interaction between parts of the mounting and its surrounding parts, tension of the supports and failure. The results of virtual tests of a car with various options for modeling mountings were compared with the results of real tests. As a result of the study, a methodology for modeling the body supports was developed, which allows providing the necessary measurement error in virtual crash test modeling.
\end{abstract}

Keywords-CAE, crash test, validation, Body-on-frame, Is-dyna, metal-rubber support.

\section{INTRODUCTION}

$\mathrm{T}$ ODAY, passive safety is one of the key properties of the car and its optimization takes a lot of time at the stage of development of the car. Trends in cheaper development costs lead to decrease in the number of full-scale tests and their replacement by virtual tests. Each full-scale passive safety test requires a new prototype, since the tested car cannot be used in the future. Using virtual models allows you to reduce development costs, but to confirm the legitimacy of using virtual tests instead of full-scale, a number of full-scale tests are necessary to validate the models. By increasing the accuracy of models for virtual tests for passive safety, it is possible to reduce the number of validation tests and achieve greater convergence of virtual tests with full-scale tests, which simplifies the refinement of the car. On the other hand, increasing the accuracy of virtual models increases the duration of calculating a virtual test, which leads to an increase in its cost. Therefore, when developing virtual models, it is necessary to find a balance between the accuracy of the results and the processor time, which takes one calculation.

The article provides a methodology for the development of virtual models of the UAZ Patriot car body supports of the 2020 model series for calculations in LS-Dyna [1] on passive safety when a car hits a deformable barrier with $40 \%$ overlap.

The vehicle under development has a body-on-frame structure. That is, the chassis parts are attached to a separate frame, consisting of stamped-welded profiles, to which the body is attached through rubber-metal supports. The body in such cars has a significantly lower stiffness than in vehicles with unibody construction, and the necessary stiffness targets are achieved due to the rigid frame. Due to this design, the main load upon hitting the barrier is assumed by the frame, which transfers them to the body through the supports. When the supports are erosed, the car structure is divided into two independent systems: frames from the chassis and the body with hinged elements and passengers. As a result of this, the body and mounted system, which has less rigidity, receives more damage than if the body carried the shock load together with the frame. Therefore, the accuracy of modeling body supports is critical in the virtual simulation of crash tests.

Fig. 1 shows an example of a body support. It consists of two elastic elements $(1,3)$, which are usually made of rubber or polyurethane, a bolt 4 that carries the entire power load, a washer 5 , nuts 6 , and sleeve 2 ensures the independence of the elastic elements and the bolt. The elastic element 1 provides the necessary elastic characteristics of the support in the vertical direction, element 3 in the radial direction. 


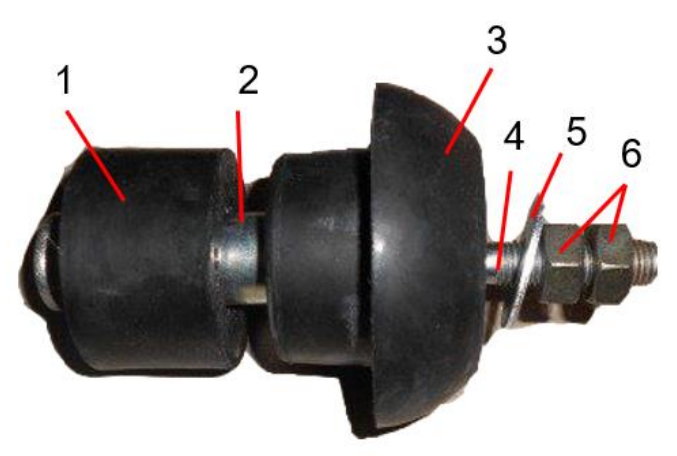

Fig. 1 Body support

As the computing power of computers increased and computer programs improved, there was a gradual complication of modeling supports [2]-[4]. In the simplest models, the frame and the body were connected using rigid ties. Such models have low accuracy, since they do not take into account the elastic and strength properties of the supports. In the most modern methods for modeling supports, high-order finite elements are used that have non-linear characteristics of materials [5], [6]. Increasing the complexity of models entails an increase in calculation time and requires more tests to obtain material characteristics. Therefore, a study was conducted on how much more detailed modeling has an impact on the accuracy of crash test calculation results in order to select a modeling method that gives acceptable accuracy without unnecessarily complicating the model and additional tests.

\section{MATERIALS AND METHODS}

A simulation was made of the impact on a deformable barrier with a $40 \%$ overlap according to the EuroNCAP rule (Fig.2). To verify the conformity of the results and determine the model of the body supports, which allows to achieve the greatest accuracy of the results, the measurements obtained as a result of a virtual crash test were compared with the measurements obtained during real tests of the same car.

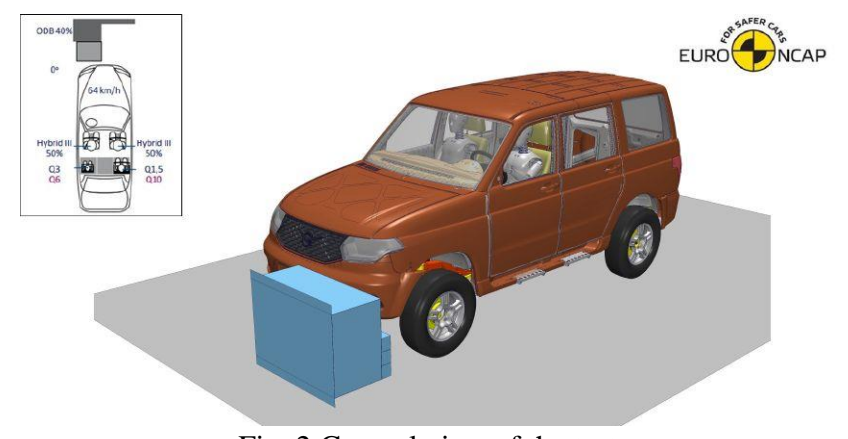

Fig. 2 General view of the test

EuroNCAP test measures a lot of data. Since the aim of this work was to develop a model of the body supports that are part of the vehicle's power structure, only data from sensors located on the power structure were studied. The most indicative of these sensors is the accelerometer installed at the base of the B-pillar. This sensor is located closest to the attachment points of the front seats and in the area where there are no high deformations associated with the impact of the car, therefore accelerations in this sensor are stable and have the greatest influence on acceleration of mannequins and a general assessment of the car according to the EuroNCAP rule.

Fig. 3 shows the acceleration graphs of the left and right Bpillars, obtained with a real impact of the car. Data was filtered using a CFC60 filter in accordance with SAE J211. Also, the graphs measured the maximum level of acceleration, valid for $3 \mathrm{~ms}$. Since maximum accelerations are unstable and vary from car to car, this integral characteristic of maximum accelerations is a convenient indicator that ensures the stability of readings.

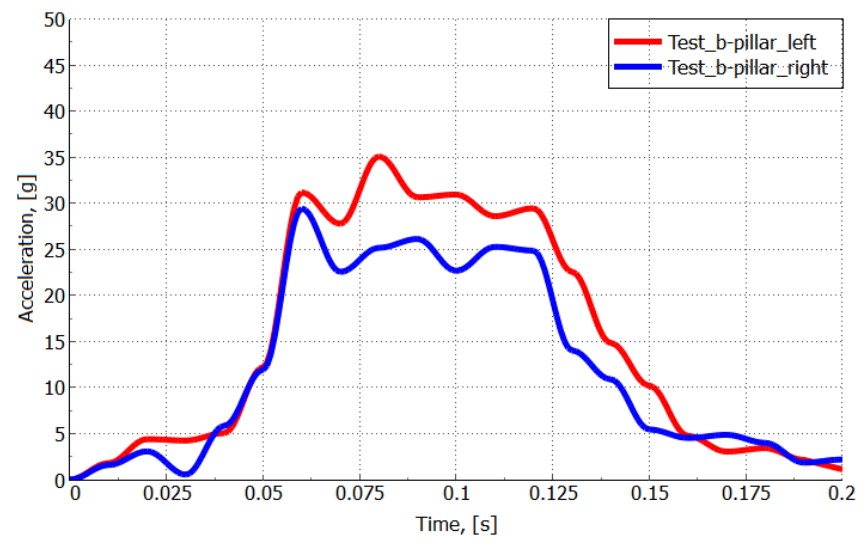

Fig. 3 Accelerations of the left and right b-pillars obtained from the test results

The simulation was carried out in the Ls-Dyna software package on 80 cores of the Tornado supercomputer [7], [8]. A complete virtual model, including a barrier and dummies, consisted of 5,000,000 elements, a car model of 3,300,000 elements. The first $200 \mathrm{~ms}$ after the start of the impact were calculated. This time is enough for all overloads in the car resulting from an impact to relax.

Several options for modeling body supports were considered:

1) Rigid connection between a body and a frame.

2) The connection between the body and the frame is ensured by beam elements and rigid connections.

3) The connection between the body and the frame is ensured by beam elements and connections with flexibility.

4) The connection between the body and the frame is ensured by beam elements with non-linear elasticity and erosion and flexibility connections.

5) The connection between the body and the frame is provided using beam elements with non-linear elasticity and erosion, solid elements of the bolt for the operation of the contact algorithm and flexibility connections.

6) The connection between the body and the frame is provided using beam elements with nonlinear elasticity and erosion, solid bolt elements for the contact algorithm and solid elements of rubber parts with nonlinear stiffness. 
When modeling bolted joints in cars during crash tests, rigid bodies like «nodal rigid body» are usually used. Since in unibody cars, as a rule, these joints are much tougher and stronger than the fastened parts, this simulation option allows us to achieve sufficiently accurate results with minimal time in calculating the model. In a body-on-frame car, the connection of the body and frame has a certain flexibility achieved through the use of rubber elements, and is extremely important in terms of strength, since the erosion of the bolt, which is the power elements connecting the body and frame, is not rare. Despite the obvious shortcomings of the support modeling option using absolutely rigid ties, it was accepted as the starting point in this study.

Fig. 4a shows the first version of the support simulation. The housing of frame 1 is connected with the sleeve in the body 2 by rigid connection 3. Gray color painted part body - floor and floor stiffer with embedded, which are made of thin shell elements with corresponding thicknesses of stamped parts. Orange color painted hinge of the frame which, like body parts, are made using shell elements.

In order to ensure the possibility of support deformation, in the second version of the simulation, beam-type elements (ELEMENT_BEAM_ELFORM_1) were added, connecting rigid bodies (NODAL_RIGID_BODY) in the body sleeve and the frame glass (painted red in Fig. 4b). For these elements, the cross-sectional characteristics of the bolt mounted in the support were set.

In the third version of the support modeling, the bolt modeled using beam elements was connected to the body using elastic elements (NODAL_RIGID_BODY was replaced by ELEMENT_DISCRETE), the stiffness characteristics, which were set in accordance with the full-scale tests of the body support stiffness assembly (Fig. 4c).

For the fourth version of the support modeling, the bolts used in the supports were tested with various deformation rates from 0.0001 units/sec to 10000 units/sec. Based on the test results, a fracture material model was created, which was later used as a material for beam-elements simulating a bolt (MAT24 MAT_PIECEWISE_LINEAR_PLASTICITY with plastic strain failure).

In previous modeling options, the elements used did not allow the Ls-Dyna contact algorithms to work effectively. Large deformations in the details of the body led to the fact that the bolt could pass through them even despite the presence of contact. For the best work of the contacts, in the fifth version of the support modeling, volume solid elements of the body of the bolt, cap and nut were added (Fig. 4d). MAT9 MAT_NULL was specified as the material of these elements. This type of material does not have rigidity and serves only for the best work of the contact algorithm. The stiffness of the bolt in this version of the simulation is still provided by beam elements. The introduction of solid-elements allows you to distribute the load from the beam elements of the bolt over the entire length of the bushings. In addition, the modeling of the cap of the bolt and nut allows real interaction between the parts to which the support is attached and the bolt - the connection is due to friction over the contact area of the cap of the bolt and nut to the washers and to the parts to which these washers fit.

Discrete-type elements do not allow to distribute the load over the entire contact area of the rubber elements to the bushings; therefore, in the sixth version of the support, these elements were replaced by solid elements imitating rubber parts (Fig. 4e). The material of these elements was selected using parametric optimization of the characteristics of the material type MAT57 MAT_MOONEY RIVLIN_RUBBER. For this, a virtual test was conducted to determine the stiffness of the support assembly. The material constants acted as parameters, and the stiffness curve of the supports obtained as a result of real tests was set as the objective function.

It is worth noting the assumptions of the models used. Since the bolt modeled by the beam element, local deformations in the bolt, stress concentration in the thread, and damage accumulation are not taken into account. When the strain reaches the value corresponding to the failure, the entire section is eroded at the same time. Also used are the average characteristics of the rubber elements as a whole, rather than the material properties. Constant damping is used. The properties of rubber elements do not take into account the strain rate.
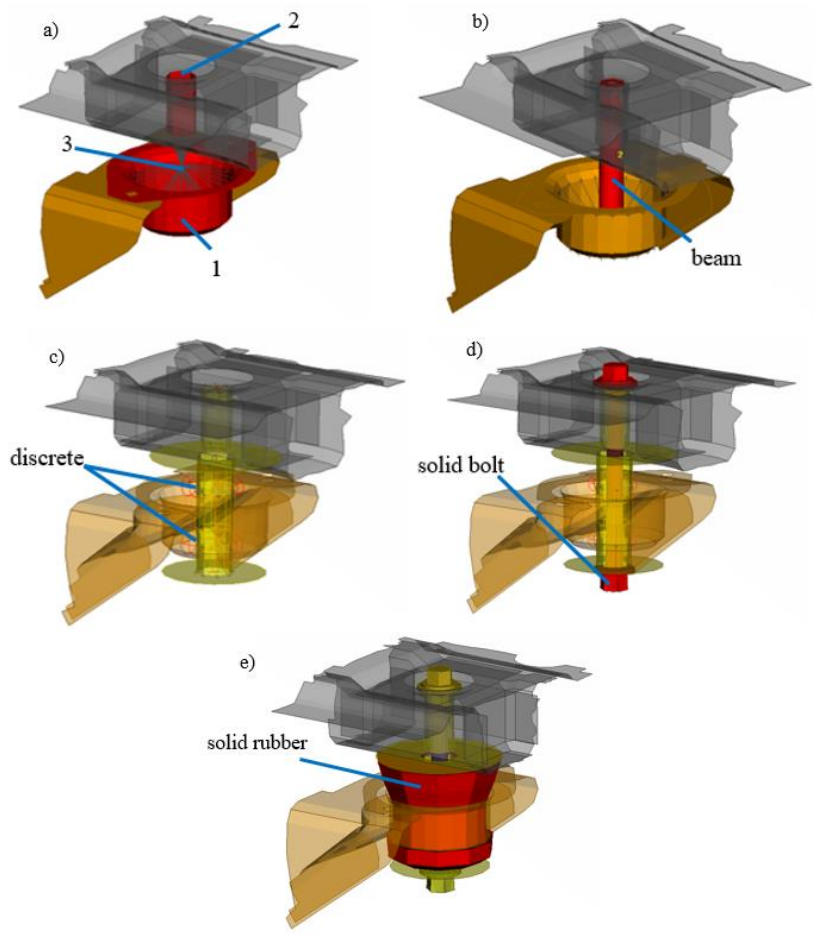

Fig. 4 Options for modeling supports

\section{RESULTS AND DISCUSSION}

The acceleration curves of the left and right B-pillar obtained as a result of virtual tests of the first version of the support modeling is shown in Fig. 5. 


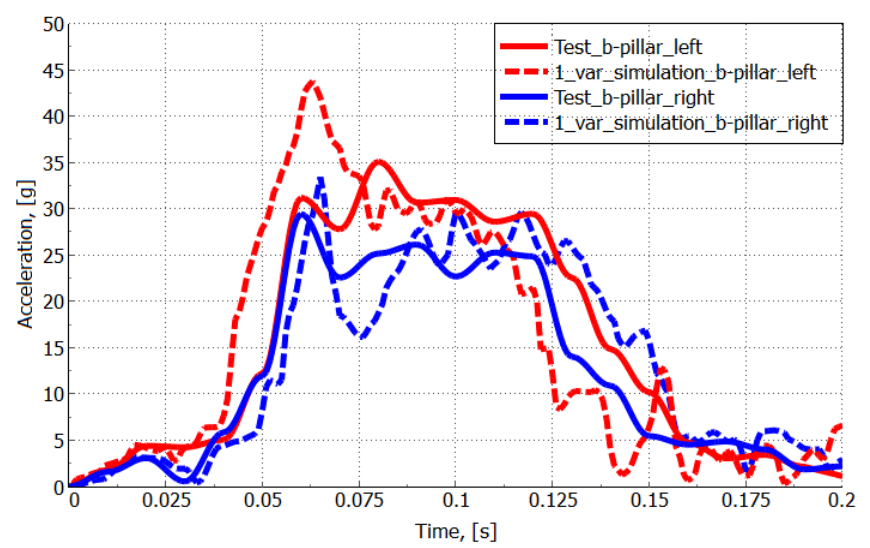

Fig. 5 Accelerations of left and right b-pillar in time from the results of virtual modeling in the first version of support modeling

You can notice from the curves that the accelerations obtained are initially conservative, but after $70 \mathrm{~ms}$ they become underestimated in comparison with real measurements. As a result, with this simulation option, the peak with maximum accelerations is missed. This is due to the fact that at $70 \mathrm{~ms}$ there is a erosion of the second left support with a real impact. In addition, the curves has a large number of oscillations. This can be attributed to the fact that the connection between the frame and the body is absolutely rigid and all vibrations of the rigid frame resulting from the impact are transmitted to the body.

The results of the calculation of accelerations in the second version of the supports, shown in Fig. 6.

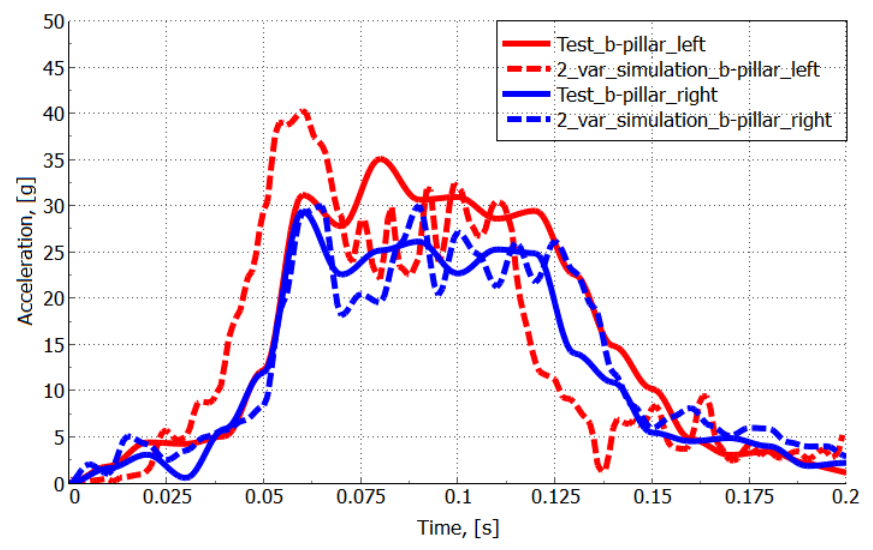

Fig. 6 Accelerations of left and right b-pillar in time from the results of virtual modeling in the second version of support modeling

According to the graphs, you can see that the peak acceleration decreased, but until the second left support failed, the results are overestimated in comparison with the real ones, and there is no maximum acceleration peak.

With the introduction of elastic elements providing the stiffness characteristics of the of the support, as in a real design, the peak acceleration decreased in comparison with the previous modeling options. In addition, multiple oscillations disappeared, and the acceleration curve became smoother. The results of the calculation of accelerations in the third version of the simulation are shown in Fig. 7.

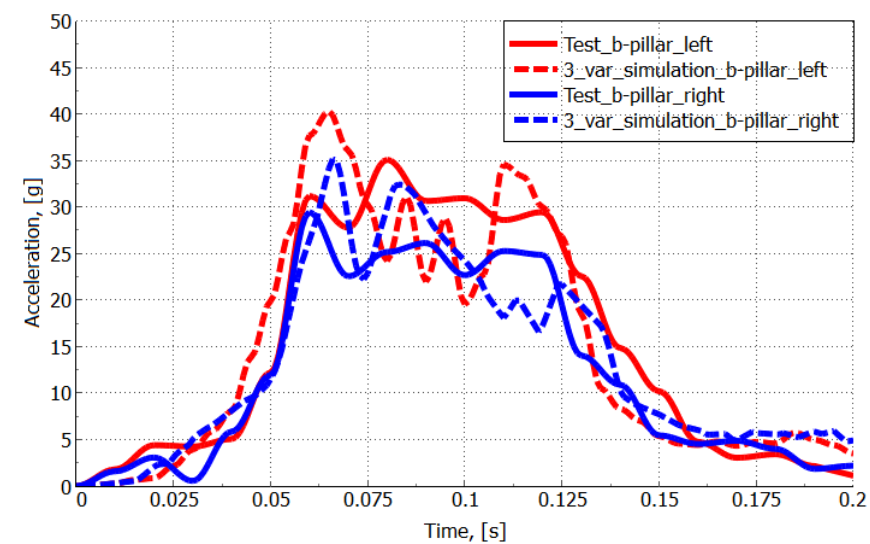

Fig. 7 Accelerations of left and right b-pillar in time from the results of virtual modeling in the third version of support modeling

A nonlinear material model with nonlinear erosion in the bolt made it possible to significantly improve the nature of the change in accelerations and bring it into line with the schedule obtained in real tests, however, the destruction of the bolt occurs somewhat earlier than in real tests. The acceleration curves of the left and right B-pillars, with this option of modeling the support is shown in Fig. 8.

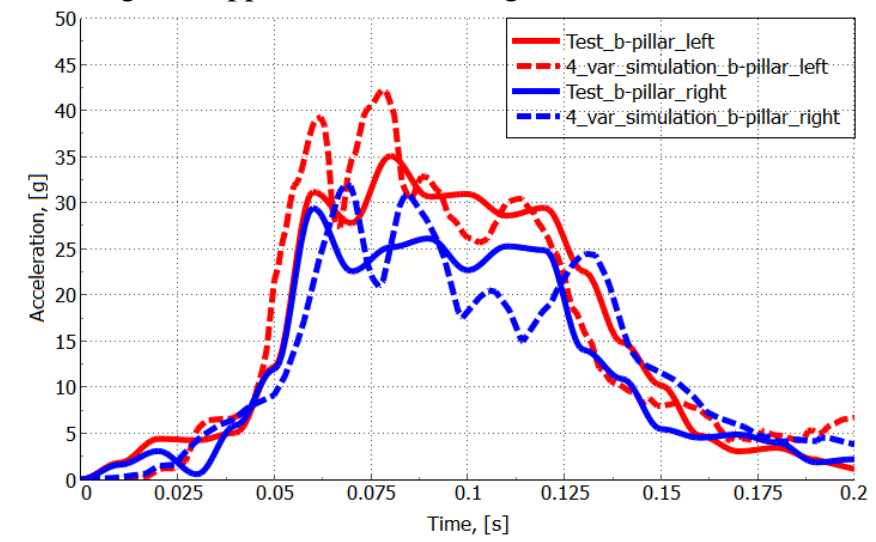

Fig. 8 Accelerations of left and right b-pillar in time from the results of virtual modeling in the fourth version of support modeling

The addition of solid elements for the contact of the bolt with the bushings made it possible to distribute the load and shift the destruction of the bolt by $70 \mathrm{~ms}$, which led to the correspondence of the moment of destruction of the bolt in the virtual model and in real tests. The acceleration curves is shown in Fig. 9. 


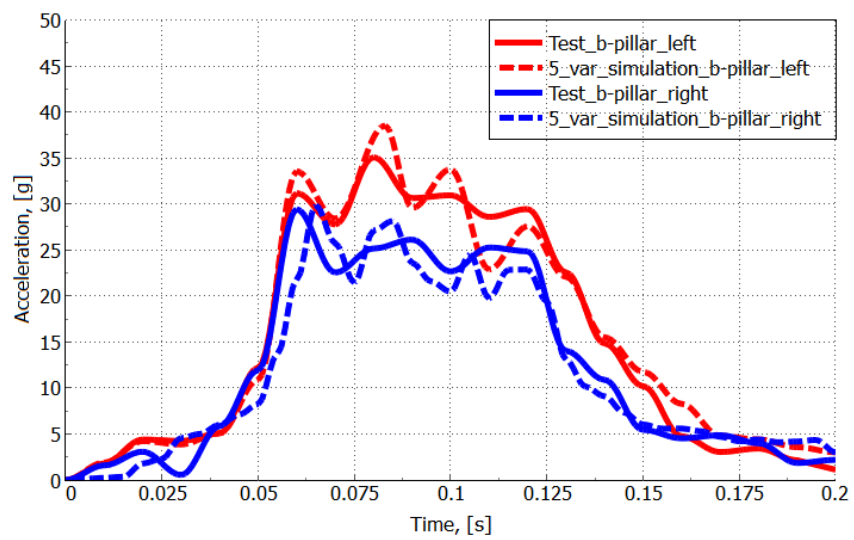

Fig. 9 Accelerations of left and right b-pillar in time from the results of virtual modeling in the fifth version of support modeling

Replacing discrete-type elastic elements with solid elements with similar stiffness characteristics did not change the nature of the accelerations but allowed to slightly improve the convergence of the results of virtual and real tests. The acceleration curves is shown in Fig. 10.

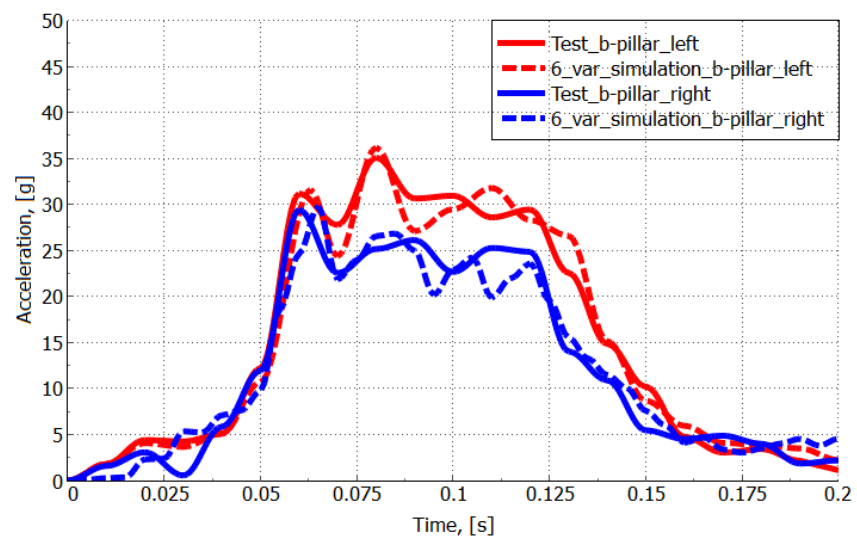

Fig. 10 Accelerations (in g) of left and right b-pillar in time from the results of virtual modeling in the sixth version of support modeling

The results of virtual tests show the best convergence with the results of field tests as the model becomes more complex and new elements are added to it. The curve of the calculation time changes as the model becomes more complex is shown in Fig. 11.

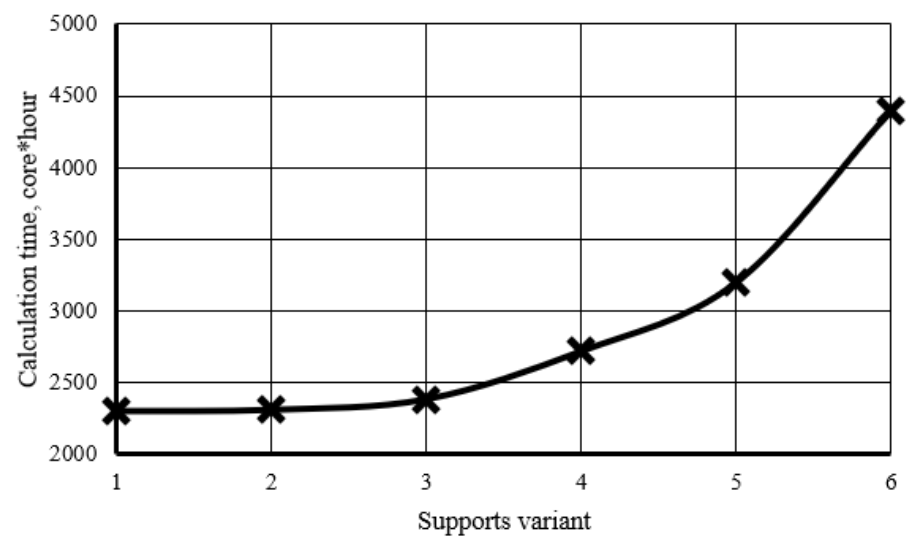

Fig 10. Calculation time vs supports variant curve
First to variant modeling adding about $5 \%$ to processor time. From the curve (Fig. 11) you can see that the addition of non-linear characteristics to the support model (variant 4 and later) gives a significant increase to the time of calculating the virtual test. By adding non-linear characteristics to the beam increased processor time by $15 \%$, adding solid bolt by another $15 \%$. The largest increase in calculation time is given by adding elements to the solid model for modeling rubber parts. Adding solid rubber increased processor time by $37 \%$ compared to the previous variant. At the same time, this modification does not significantly increase the accuracy of the calculation. When a solid bolt is added to the model, the error in maximum accelerations is within $10 \%$ and is conservative. Moreover, the nature of the curve coincides with real tests. This is an acceptable level of error and allows you to judge the behavior of the car during crash tests. Therefore, it was decided to use the fifth version of modeling the body supports in further calculations of crash tests of frame cars.

This modeling methodology was also used to simulate crash tests of UAZ Patriot of previous model years and showed a similar similarity with the results of real tests, which allows us to speak about its reliability. See also [9] and [10].

\section{CONCLUSION}

As a result of the study, a methodology was developed for modeling the body supports of body-on-frame cars for virtual modeling crash tests. The body acceleration obtained as a result of the calculations were compared with the same measures obtained during real tests. The technique was developed based on the accuracy of the results and the calculation time. The selected variant, including erosion of the bolt, the elastic characteristics of the rubber, the contact interaction, friction forces, is the optimal compromise between accuracy and calculation time.

Due to the lack of the possibility of fracture and deformation, the previously used models of supports (variants 1 and 2) could show a high error in the measurement of accelerations (more than 20\%) .Application of a more complex support model (option 5) allows to reduce the error in the measurement of accelerations to no more than $10 \%$, the error in the displacement and determination of the assessment according to the EuroNCAP protocol in the virtual test compared to the real one does not exceed 5\%.

\section{ACKNOWLEDGMENT}

The study is done by the head contractor of R\&D SPbPU with the financial support of the Ministry of science and higher education of the Russian Federation in the framework of grant No. 075-11-2018-017 from "03" March 2017 (the internal contract number 03.G25.31.0233). 


\section{References}

[1] LS-DYNA Theory manual, Livermore Software Technology Corporation, Livermore, California, 2006.

[2] J. Cheng, "Body Mount Crash Characteristic Identification - Test and Analysis.", SAE Transactions, vol. 103, pp. 403-410, 1994.

[3] Park, Sae U., Madhu R. Koka, Kevin R. Thomson, Jeffrey L. Robbins, and Venkata Nambori. "A Study on Vehicle Elastomer Mount Preloading and Impact Response with Test Validation." SAE Transactions, vol. 114, pp. 713719, 2005.

[4] Y. Chen, T. Tyan and O. Faruque, "Dynamic Testing and CAE Modeling of Body Mount An Application in the Frontal Impact Analysis of a Body-on-Frame Vehicle", SAE Technical Paper Series, 2003.

[5] V. Sandell, "Extraction of Material Parameters for Static and Dynamic Modeling of Carbon Black Filled Natural Rubbers", master's level, Luleå University of Technology, 2017.

[6] J. Oscar and G. Centeno, "FINITE ELEMENT MODELING OF RUBBER BUSHING FOR CRASH SIMULATION Experimental Tests and Validation", Master's dissertation, Lund University, 2009.

[7] Belytschko T., Liu W.K. and Moran B., Nonlinear Finite Elements for Continua and Structures. Wiley, Chichester, U.K. 2000.

[8] Cook R.D., Malkus D.S. and Plesha M.E., Concepts and Applications of Finite Element Analysis. John Wiley \& Sons, 1989.

[9] Ioan Enescu, Some Researches Regarding Stress Intensity Factors in Crack Closure Problems, WSEAS Transactions on Applied and Theoretical Mechanics, pp. 187-192 Volume 13, 2018

[10] Pavel Padevet, Tereza Otcovska, Barbora Muzikova, Material Properties of Rammed Earth Prepared from Illitic-Kaolinitic Clay, WSEAS Transactions on Applied and Theoretical Mechanics, Volume 13, 2018, pp. 181186 


\section{Creative Commons Attribution License 4.0 \\ (Attribution 4.0 International, CC BY 4.0)}

This article is published under the terms of the Creative Commons Attribution License 4.0

https://creativecommons.org/licenses/by/4.0/deed.en_US 